\title{
The Sun's journey through the local interstellar medium: the paleoLISM and paleoheliosphere
}

\author{
P. C. Frisch ${ }^{1}$ and J. D. Slavin ${ }^{2}$ \\ ${ }^{1}$ University of Chicago, Department of Astronomy and Astrophysics, 5640 S. Ellis Ave., Chicago, IL 60637, USA \\ ${ }^{2}$ Harvard-Smithsonian Center for Astrophysics, 60 Garden Street, Cambridge, MA 02138, USA
}

Received: 12 April 2006 - Revised: 30 June 2006 - Accepted: 30 June 2006 - Published: 8 August 2006

\begin{abstract}
Over the recent past, the galactic environment of the Sun has differed substantially from today. Sometime within the past $\sim 130000$ years, and possibly as recent as $\sim 56000$ years ago, the Sun entered the tenuous tepid partially ionized interstellar material now flowing past the Sun. Prior to that, the Sun was in the low density interior of the Local Bubble. As the Sun entered the local ISM flow, we passed briefly through an interface region of some type. The low column densities of the cloud now surrounding the solar system indicate that heliosphere boundary conditions will vary from opacity considerations alone as the Sun moves through the cloud. These variations in the interstellar material surrounding the Sun affected the paleoheliosphere.
\end{abstract}

\section{Introduction}

The boundary conditions of the heliosphere at any given point in its history are set by the interstellar cloud that happened to surround the Solar System at that time. Our variable galactic environment affects the physical properties of the heliosphere, and the fluxes of galactic cosmic rays and interstellar byproducts reaching the Earth. The work of Hans Fahr has, from the earliest until now, provided a strong foundation for our understanding of the effect of the ISM on the heliosphere and on Earth (e.g. Fahr, 1968, 1974; Ripken and Fahr, 1983; Scherer and Fahr, 2003; Yeghikyan and Fahr, 2004).

The properties of both astrospheres and the heliosphere are highly responsive to the boundary conditions supplied by the interstellar medium and the interstellar radiation field (Fahr, 1978; Frisch, 1993, 1997; Zank and Frisch, 1999; Scherer et al., 2002; Florinski et al., 2003b; Mueller et al., 2006; Frisch, 2006). The space motion of the Sun, when compared to the column densities of interstellar material (ISM) towards

Correspondence to: P. Frisch

(frisch@oddjob.uchicago.edu) stars near the Sun, indicates that sometime in the late Quaternary the Sun, which has been moving through the very low density region known as the Local Bubble, encountered the cluster of local interstellar clouds (CLIC) flowing away from the direction of the Scorpius-Centaurus Association (Frisch, 1981, 1997; Frisch and York, 1986; Frisch and Slavin, 2006, FS06). Mediating the interaction between the very low density Local Bubble and the tepid CLIC will be a thin interface of some type (Slavin, 1989; Slavin and Frisch, 2002).

Regardless of the Local Bubble plasma pressure, the large contrast $\left(>10^{3}\right)$ between neutral ISM densities in the Local Bubble versus the CLIC must have generated significant changes in the heliosphere, because pickup ions and anomalous cosmic rays are processed interstellar neutrals. The well-known anticorrelation between galactic cosmic ray fluxes at Earth and the solar magnetic activity cycle, mediated by the heliosphere, suggests that the transition between the Local Bubble cavity and CLIC altered both the heliosphere and the galactic cosmic ray flux at Earth, with possible implications for the terrestrial climate. Heliosphere models have now been constructed for a range of interstellar boundary conditions (e.g. Mueller et al., 2006; Florinski et al., 2003a; Scherer, 2000; Yeghikyan and Fahr, 2004). The models show that the ISM surrounding the Sun during the geological past (or the "paleoLISM") did affect the heliosphere during the geological past (or the "paleoheliosphere"), and therefore also the galactic cosmic ray flux at Earth.

In this paper, we postulate a geometric model for the CLIC, supplemented by equilibrium models that provide the cloud density. The entry epoch of the Sun into the CLIC (also known as the Local Fluff) depends on the relative Sun-CLIC velocities and the distribution of the CLIC gas. To estimate this transition epoch, the distribution of CLIC gas is derived from a simple model of the cloud morphology using data for $\mathrm{H}^{\circ}$ and $\mathrm{D}^{\circ}$ towards nearby stars (Sect. 2.1), combined with the CLIC density found from photoionization equilibrium models of nearby ISM (Sect. 2.2). The entry of the Sun

Published by Copernicus GmbH on behalf of the Arbeitsgemeinschaft Extraterrestrische Forschung e.V. 
Table 1. HC and LSR Velocities of the Sun, CLIC, and LIC.

\begin{tabular}{lccc}
\hline & $\begin{array}{c}\text { Sun } \\
\text { Motion }\end{array}$ & $\begin{array}{c}\text { Upwind } \\
\text { CLIC }\end{array}$ & $\begin{array}{c}\text { Upwind } \\
\text { LIC }\end{array}$ \\
\hline $\mathrm{HC}:$ & & & \\
$\mathrm{V}(\mathrm{km} / \mathrm{s})$ & - & $-28.1 \pm 4.6$ & $-26.3 \pm 0.4$ \\
$\ell, b$ & - & $12.4^{\circ}, 11.6^{\circ}$ & $3.3^{\circ}, 15.9^{\circ}$ \\
$\mathrm{LSR}$ & & \\
$\mathrm{V}(\mathrm{km} / \mathrm{s})$ & 19.5 & -19.4 & -20.7 \\
$\ell, b$ & $56^{\circ}, 23^{\circ}$ & $331.0^{\circ},-5.1^{\circ}$ & $317.8^{\circ},-0.5^{\circ}$ \\
$\mathrm{LSR}$ & & & \\
$\mathrm{V}(\mathrm{km} / \mathrm{s})$ & 13.4 & -17.0 & -15.7 \\
$\ell, b$ & $27.7^{\circ}, 32.4^{\circ}$ & $2.3^{\circ},-5.1^{\circ}$ & $346.0^{\circ}, 0.1^{\circ}$ \\
\hline
\end{tabular}

Note: The Standard solar motion, $\mathrm{LSR}_{\mathrm{Std}}$, corresponds to a velocity of $19.5 \mathrm{~km} / \mathrm{s}$ towards $\ell=56^{\circ}, b=23^{\circ}$. The Hipparcos solar motion, $\mathrm{LSR}_{\mathrm{Hip}}$, corresponds to a velocity of $13.4 \mathrm{~km} / \mathrm{s}$ towards $\ell=27.7^{\circ}$, $b=32.4^{\circ}$ (Dehnen and Binney, 1998).

into the CLIC is then found from the cloudlet velocities in the downwind direction (Sect. 2.1). Prior to encountering the CLIC, the Sun was in the very low density gas of the Local Bubble interior for several million years (Sect. 3). The limitations of the simple assumptions underlying these estimates are discussed briefly in Sect. 4 .

\section{Sun passage into very local ISM}

The epoch when the Sun first encountered the CLIC gas can be estimated from data on ISM column densities and velocities towards nearby stars, combined with theoretical models that provide the average cloud density. These photoionization models provide a second important result about the paleoheliosphere, by showing clearly that the boundary conditions of the heliosphere, in particular the ionization level of hydrogen and the electron density, vary from radiative transfer effects alone as the Sun traverses low opacity ISM (Sect. 2.2, Slavin and Frisch, 2002, SF02,SF06).

\subsection{Local ISM data and distribution}

The Sun has recently entered the CLIC, which itself is inhomogeneous. To estimate the entry date, we map the CLIC gas by assuming that the distances to the CLIC edges are given by $N\left(\mathrm{H}^{\circ}\right) /\left\langle n\left(\mathrm{H}^{\circ}\right)\right\rangle$, where $N\left(\mathrm{H}^{\circ}\right)$ are column densites towards nearby stars. The average space density $\left\langle n\left(\mathrm{H}^{\circ}\right)\right\rangle$ is provided by radiative transfer models (Sect. 2.2).

CLIC dynamics indicate an origin related to a superbubble caused by star evolution in the Scorpius-Ophiuchus Association (Frisch, 1981). The bulk flow velocity vector of the CLIC gas past the Sun is $\sim-28 \pm 4.6 \mathrm{~km} / \mathrm{s}$, from the direction $\ell, b \sim 12^{\circ}, 12^{\circ}$ in heliocentric coordinates (HC, Frisch et al., 2002). This vector is based on absorption components towards $\sim 60$ stars, obtained at resolutions of $0.3-3.0 \mathrm{~km} / \mathrm{s}$. Most of the Hyades stars were excluded from the star sample underlying this vector, because ISM with a poorly defined relationship to the CLIC is found inside of this cluster (Redfield and Linsky, 2001). The LIC is the best understood member of the CLIC, with a precisely known velocity based on Ulysses $\mathrm{He}^{\circ}$ data of $-26.3 \pm 0.4 \mathrm{~km} / \mathrm{s}$ from $\ell, b=3.3^{\circ}, 15.9^{\circ}$ (HC, Witte, 2004). The exact value for the CLIC bulk flow depends on the underlying star sample, since the flow gradually decelerates towards the downwind direction (FS06). Examples of the deceleration are the LIC, found in the downwind direction at $+26 \mathrm{~km} / \mathrm{s}$, and the Apex cloud, found at $-35 \mathrm{~km} / \mathrm{s}$ in the upwind direction (HC).

The velocity vectors of the CLIC, LIC, and the Sun are listed in Table 1 in both HC coordinates and the local standard of rest (LSR) velocity frame, for both the Standard and Hipparcos-based LSR frame. The uncertainty in the LSR occurs because the age distributions of the star samples underlying these two LSR vectors were different (Dehnen and Binney, 1998; Mihalas and Binney, 1981).

Data on $N\left(\mathrm{H}^{\mathrm{o}}\right)$ are drawn from observations of $\mathrm{D}^{\mathrm{o}}$ and $\mathrm{H}^{\mathrm{o}}$ towards nearby cool and hot stars (Wood et al., 2005; Redfield and Linsky, 2004a; Frisch et al., 2002), white dwarf stars (Wolff et al., 1999; Lehner et al., 2003; Vallerga, 1996; Oliveira et al., 2003; Hébrard and Moos, 2003; Wood et al., 2002; Lemoine et al., 2002; Kruk et al., 2002; Frisch, 1995), and nearby stars with observations of interstellar $\mathrm{Ca}^{+}$(e.g. Frisch et al., 2002; Crawford et al., 1998; Crawford and Dunkin, 1995, and references therein). The column densities of all velocity components towards a star are summed to obtain the total $N\left(\mathrm{H}^{\mathrm{o}}\right)$ towards the CLIC surface. For stars with $\mathrm{D}^{\mathrm{o}}$ data, a conversion factor of $N\left(\mathrm{H}^{\circ}\right) / N\left(\mathrm{D}^{\mathrm{o}}\right)=1.5 \times 10^{-5}$ is used. The total $\mathrm{H}^{\mathrm{o}}$ column densities to the CLIC surface are in the range $N\left(\mathrm{H}^{\mathrm{o}}\right)=0.3 \times 10^{18}$ to $\sim 10^{19} \mathrm{~cm}^{-2}$.

Optical $\mathrm{Ca}^{+}$data are also used, however the ratio $N\left(\mathrm{Ca}^{+}\right) / N\left(\mathrm{H}^{\circ}\right)$ is highly variable. In cold clouds $\sim 99.7 \%$ of the $\mathrm{Ca}$ is depleted onto dust grains. Both depletion and ionization affect $\mathrm{Ca}^{+}$in warm clouds such as the CLIC; for example $\mathrm{Ca}^{++} / \mathrm{Ca}^{+}>1$ if $T>4000 \mathrm{~K}$ and $n(\mathrm{e})<0.13 \mathrm{~cm}^{-3}$ (e.g. Welty et al., 1996). The ratio $N\left(\mathrm{H}^{\mathrm{o}}\right) / N\left(\mathrm{Ca}^{+}\right)=10^{-8}$ is used, based on the three absorption components observed in both $\mathrm{Ca}^{+}$and $\mathrm{H}^{\circ}$ towards $\alpha$ Aql $\left(d=5 \mathrm{pc}, \ell, b \sim 48^{\circ},-9^{\circ}\right.$, e.g. Frisch et al., 2002; Redfield and Linsky, 2004a; Ferlet et al., 1986).

Looking only at stars within $10 \mathrm{pc}$, average values of $\left\langle N\left(\mathrm{H}^{\circ}\right)\right\rangle \sim 10^{18} \mathrm{~cm}^{-2}$ and $\left\langle n\left(\mathrm{H}^{\circ}\right)\right\rangle \sim 0.07 \mathrm{~cm}^{-3}$ are found. Based on temperature and turbulence data in Redfield and Linsky (2004b), objects within $10 \mathrm{pc}$ show a temperature and turbulence range of $T=1700-12600 \mathrm{~K}$ and $\xi=0-5.5 \mathrm{~km} / \mathrm{s}$, with mean values $6740 \pm 2800 \mathrm{~K}$ and $\xi=1.9 \pm 1.0 \mathrm{~km} / \mathrm{s}$. The $\xi$ variable represents deviations from a Maxwellian velocity distribution for the atoms contributing to the absorption line components, and as such is a mock turbulence that includes unresolved clouds. 


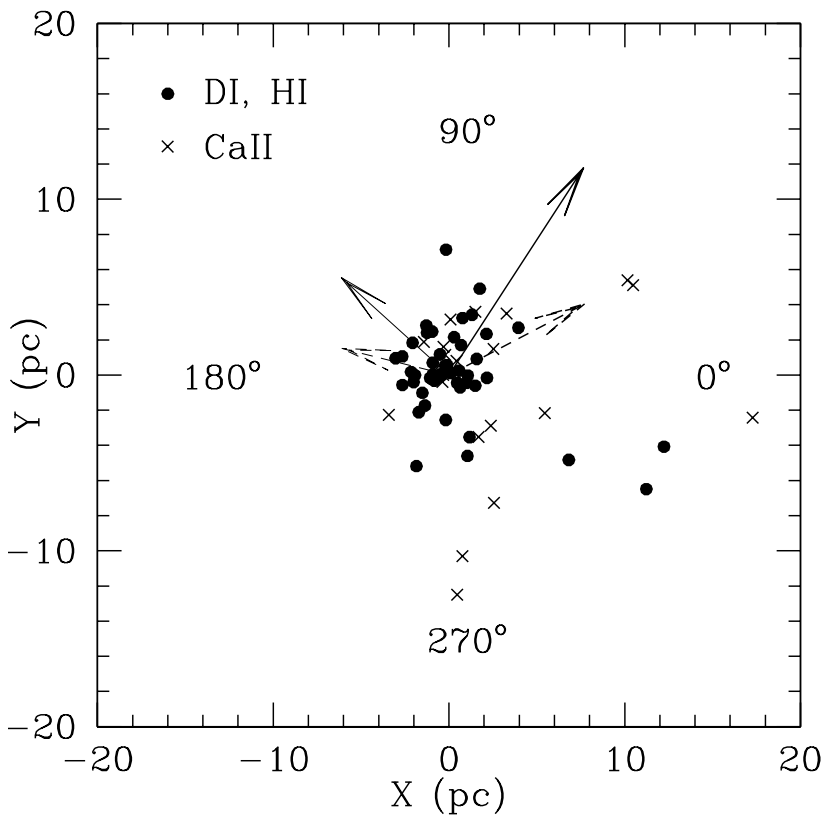

Fig. 1. The distances to the edge of the CLIC, projected onto the galactic plane, as calculated from $N\left(\mathrm{H}^{\mathrm{O}}\right) /\left\langle n\left(\mathrm{H}^{\circ}\right)\right\rangle$ for stars near the Sun. Distances are based on observations of $\mathrm{H}^{\mathrm{O}}$ (dots), $\mathrm{D}^{\mathrm{O}}$ (dots), and $\mathrm{Ca}^{+}$(crosses) (see text). The directions $\ell=0^{\circ}, 90^{\circ}, 180^{\circ}$, and $270^{\circ}$ are labeled. The arrows directed to the left show the CLIC motion through the Local Standard of Rest (LSR) based on the standard (solid) and Hipparcos (dashed) solar apex motions, while the arrows to the right show the motion of the Sun through the LSR based on the Hipparcos and Standard apex values (Table 1).

The range of temperatures inferred for the CLIC, combined with the macroturbulence of $\pm 4.6 \mathrm{~km} / \mathrm{s}$, show that the CLIC is inhomogeneous and that the boundary conditions of the heliosphere will vary during the next thousands of years as the Sun traverses the CLIC.

The CLIC is fully contained in the nearest $\sim 35 \mathrm{pc}$ of space. The distances to the edges of the CLIC are shown in Figs. 1 and 2. These figures are constructed from the data listed above, and assuming $\left\langle n\left(\mathrm{H}^{\circ}\right)\right\rangle=0.17 \mathrm{~cm}^{-3}$ (discussed in Sect. 2.2). This ISM distribution is assumed to contain no gaps.

An extended dilute HII region found towards $\lambda$ Sco (York, 1983) may contribute to the excess cloud length towards the galactic center that is indicated by $\mathrm{Ca}^{+}$data. Highly ionized gas at CLIC velocities is found towards both $\lambda$ Sco $\left(\ell, b=352^{\circ},-2^{\circ}\right)$ and HD 149499B $(d=37 \mathrm{pc}$, $\ell, b=330^{\circ},-7^{\circ}$ ), where for instance $\mathrm{N}^{+} / \mathrm{N}^{\circ}=1.9$ (Lehner et al., 2003).

\subsection{Boundary condition variations from cloud opacity}

An important influence on the heliosphere while the Sun traverses the low opacity CLIC is the change in helio-

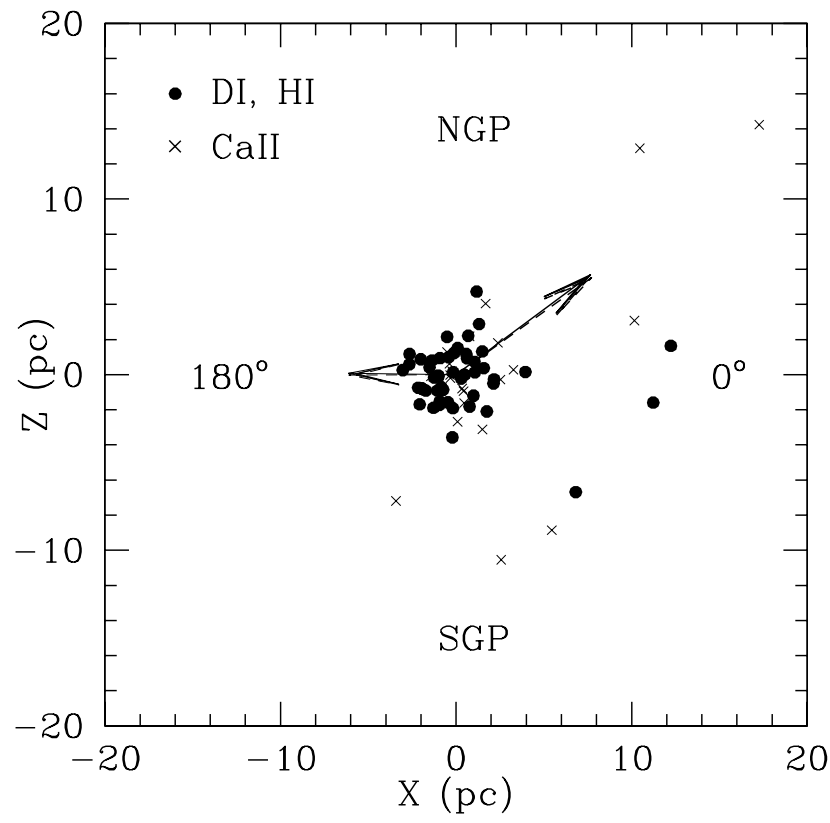

Fig. 2. Same as Fig. 1, except that the projection is on a meridian plane perpendicular to the galactic plane.

spheric boundary conditions caused by ionization variations due to the attenuation of photons with energy $E>13.6 \mathrm{eV}$ (the ionization edge of $\mathrm{H}^{\mathrm{o}}$ ). These variations are shown by our radiative transfer (RT) models (Slavin and Frisch, $2002)^{1}$. The CLIC is partially opaque to H-ionizing photons $(\lambda<912 \AA)$ and nearly transparent to He-ionizing photons $(\lambda<504 \AA)$. A cloud optical depth of $\tau \sim 1$ is achieved for $\log N\left(\mathrm{H}^{\mathrm{o}}\right) \sim 17.2 \mathrm{~cm}^{-2}$ and $\sim 17.7 \mathrm{~cm}^{-2}$, respectively, at the $\mathrm{H}^{\mathrm{O}}$ and $\mathrm{He}^{\circ}$ ionization edges.

A series of models have been constructed to study these opacity effects (SF02, SF06). These models employ the CLOUDY radiative transfer code, which incorporates a wide range of physical processes to model a cloud under the conditions of photoionization equilibrium (Ferland et al., 1998). The models are constrained by observations of the CLIC towards $\epsilon \mathrm{CMa}$ (Gry and Jenkins, 2001), observations of pickup ions, anomalous cosmic rays, and $n\left(\mathrm{He}^{\circ}\right)$ inside of the solar system, and interstellar radiation field data and models. The radiation field is based on radiation sources affecting the solar environment (see SF02), and extends out to soft X-ray energies (Sect. 3.2). Figure 3 summarizes the variations in neutral densities that are obtained for equilibrium calculations of low density ISM similar to the LIC (see SF02 and

\footnotetext{
${ }^{1}$ By "radiative transfer effects", we mean that the spectrum of the radiation field, including both point source and diffuse contributions, is substantially modified for energies $E>13.6 \mathrm{eV}$ as the radiation penetrates more deeply into the cloud. This is the case for the CLIC gas, as shown by the relative opacities of the cloud to $\mathrm{H}-$ versus He- ionizing photons.
} 


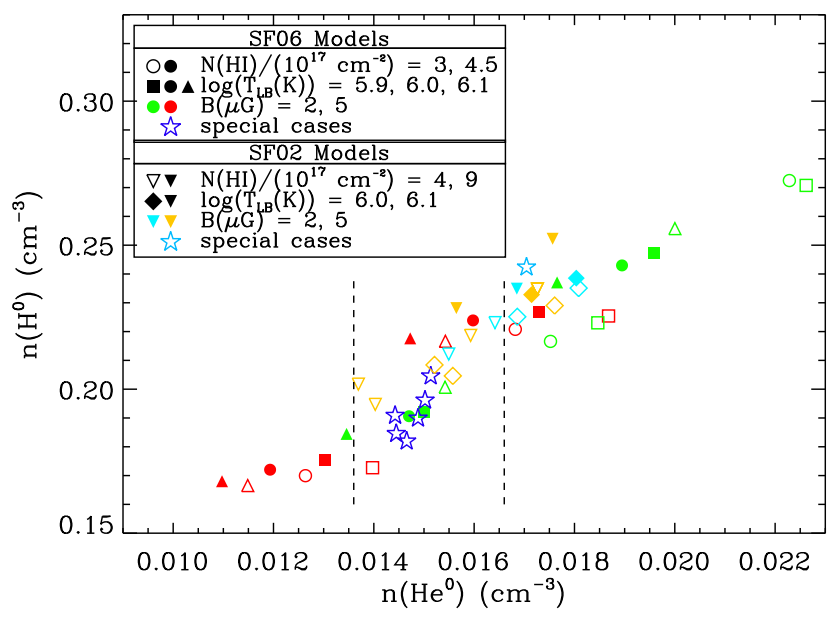

Fig. 3. The densities of $\mathrm{H}^{\mathrm{o}}$ and $\mathrm{He}^{\circ}$, as predicted by radiative transfer models of the CLIC, are shown for a range of equilibrium conditions based on models in SF02 and SF06. The symbols give the model parameters: symbol colors indicate the magnetic field strength in the cloud interface; symbol fill gives gives the assumed HI column density; symbol shape gives the assumed temperature of the Local Bubble plasma. The stars are special parameter sets which do not fall on the grid of model parameters, but rather are chosen to better match the data. The range of current values for $n\left(\mathrm{He}^{\circ}\right)$, based on Ulysses and pickup ion data, are shown as vertical lines (Möbius et al., 2004). Although a range of $n\left(\mathrm{H}^{\circ}\right)$ values are consistent with $n\left(\mathrm{He}^{\circ}\right)$, the radiative transfer models providing the best agreement with ISM observations both inside of the heliosphere and towards nearby stars give $n\left(\mathrm{H}^{\circ}\right)=0.18-0.21 \mathrm{~cm}^{-3}$.

SF06). The best of these models (models 2 and 8 in SF02) give $n\left(\mathrm{H}^{\circ}\right)=0.18-0.21 \mathrm{~cm}^{-3}$ and $n\left(\mathrm{H}^{+}\right)=0.1 \mathrm{~cm}^{-3}$ at the solar location. These model results that best predict the observed gas densities, for a study still in progress, give a mean value of $\left\langle n\left(\mathrm{H}^{\circ}\right)\right\rangle \sim 0.17 \mathrm{~cm}^{-3}$ for the sightline towards the downwind cloud surface.

Figure 4 shows the effects of cloud opacity. The ionization levels of $\mathrm{H}, \mathrm{O}$, and $\mathrm{N}$, which are coupled by charge exchange, decrease by $\geq 20 \%$ from the cloud exterior to the solar location. $^{2}$ In contrast, $\mathrm{He}$ and $\mathrm{Ne}$ ionizations, which require photons more energetic by $>50 \%$, vary little. Guesstimates indicate that for $\sim 50 \%$ filtration of $\mathrm{H}^{\mathrm{o}}$, converting $20 \%$ of the $\mathrm{H}$ from $\mathrm{H}^{\mathrm{o}}$ to $\mathrm{H}^{+}$would raise the $\mathrm{H}$ pressure confining the paleoheliosphere at the cloud surface on the order of $\sim 10 \%$, as compared to the present-day value. The timescales over which these variations occur depend on the assumed cloud shape, and may be as short as thousands of years.

Ionization variations that are five times larger than those we have modeled are observed in the very local ISM. Warm partially ionized material (WPIM, $T>5000 \mathrm{~K}$ ) such as the CLIC is widespread near the Sun, and is sampled

\footnotetext{
${ }^{2}$ The small decrease in $\mathrm{N}^{\circ} / \mathrm{H}^{\mathrm{o}}$ at cloud edge results from a high $\mathrm{N}^{\circ}$ photoionization cross-section.
}

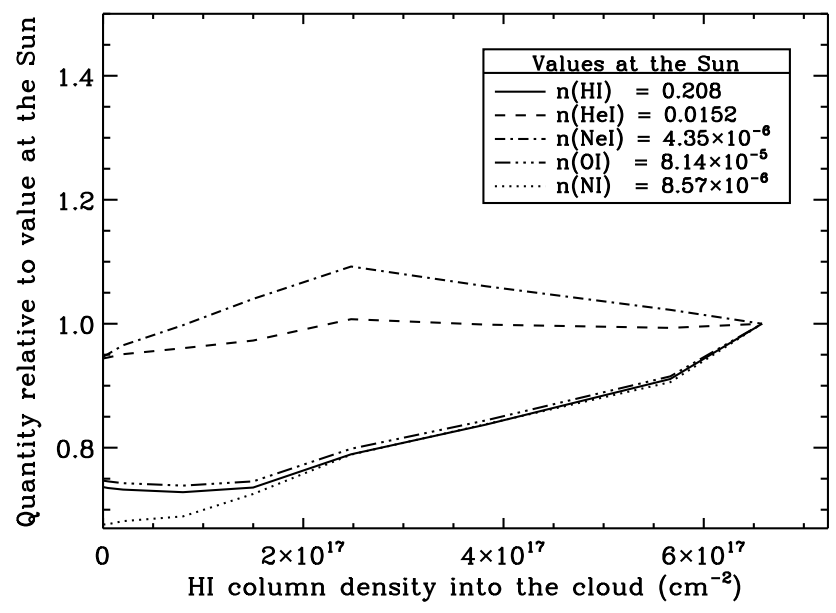

Fig. 4. Variation of neutral densities, due to radiative transfer effects between the Sun and cloud surface, for Model 2 from SF02. Shown are variations in neutral densities between the Sun $\left(N\left(\mathrm{H}^{\mathrm{o}}\right)=6.5 \times 10^{17} \mathrm{~cm}^{-2}\right)$ and cloud surface $\left(N\left(\mathrm{H}^{\mathrm{o}}\right)=0\right)$ for $\mathrm{H}^{\mathrm{o}}$, $\mathrm{He}^{\circ}, \mathrm{Ne}^{\circ}, \mathrm{O}^{\circ}$, and $\mathrm{N}^{\circ}$. At the heliopause, $n\left(\mathrm{He}^{\circ}\right) \sim 0.015 \mathrm{~cm}^{-3}$, $n\left(\mathrm{H}^{\circ}\right) \sim 0.21 \mathrm{~cm}^{-3}, n(\mathrm{e}) \sim 0.1 \mathrm{~cm}^{-3}$. The cloud surface is at the left, and the solar location is at the right of the figure.

by data on $\mathrm{N}^{+}$and $\mathrm{N}^{\circ}$ from Copernicus and FUSE. Values of $N\left(\mathrm{~N}^{+}\right) / N\left(\mathrm{~N}^{\circ}\right) \sim 0.4-2.0$ are typical for low density sightlines, $N\left(\mathrm{H}^{\mathrm{o}}\right)<10^{19} \mathrm{~cm}^{-2}$ (e.g. Rogerson et al., 1973; Oliveira et al., 2003; Lehner et al., 2003). The best CLIC models give $n\left(\mathrm{~N}^{+}\right) / n\left(\mathrm{~N}^{\circ}\right) \sim 0.76$ at the Sun (nos. 2 and 8 in SF02). Consequently, as the CLIC sweeps past the Sun variations larger than $10 \%$ in the heliosphere may be caused by variable interstellar ionizations. The $\mathrm{H}^{+}$gas towards $\lambda$ Sco and HD 149499B, $37 \mathrm{pc}$ away in the upwind direction, is an example of an ionized cloud that could engulf the Sun in the next million years.

\subsection{Solar encounter with the CLIC}

When did the Sun enter the CLIC? With the exception of the LIC, only the Doppler-shifted radial components of cloud motions are observed. Hence only approximate estimates of the epoch that the Sun made the transition from the very low density Local Bubble to the CLIC are possible. We get an averaged value for the time of encounter by calculating the distance to the CLIC edge for all stars within $50 \mathrm{pc}$, and between $\ell=170^{\circ} \pm 30^{\circ}$ and $|b|<30^{\circ}$, and comparing this distance and the HC ISM velocity towards each star. The distance to the cloud edge is given by $N\left(\mathrm{H}^{\circ}\right) /\left\langle n\left(\mathrm{H}^{\circ}\right)\right\rangle$, where $\left\langle n\left(\mathrm{H}^{\circ}\right)\right\rangle=0.17 \mathrm{~cm}^{-3}$. Nine stars in Figs. 1 and 2 fall in this interval. The average distance to the cloud edges for these stars is $2.8 \mathrm{pc}$ (range $1.2-3.8 \mathrm{pc}$ ), and the average entry time of the Sun into the CLIC is 120000 years ago (range 56000200000 years ago). Restricting the downwind stars to those within 30 parsecs yields four stars, and does not significantly 


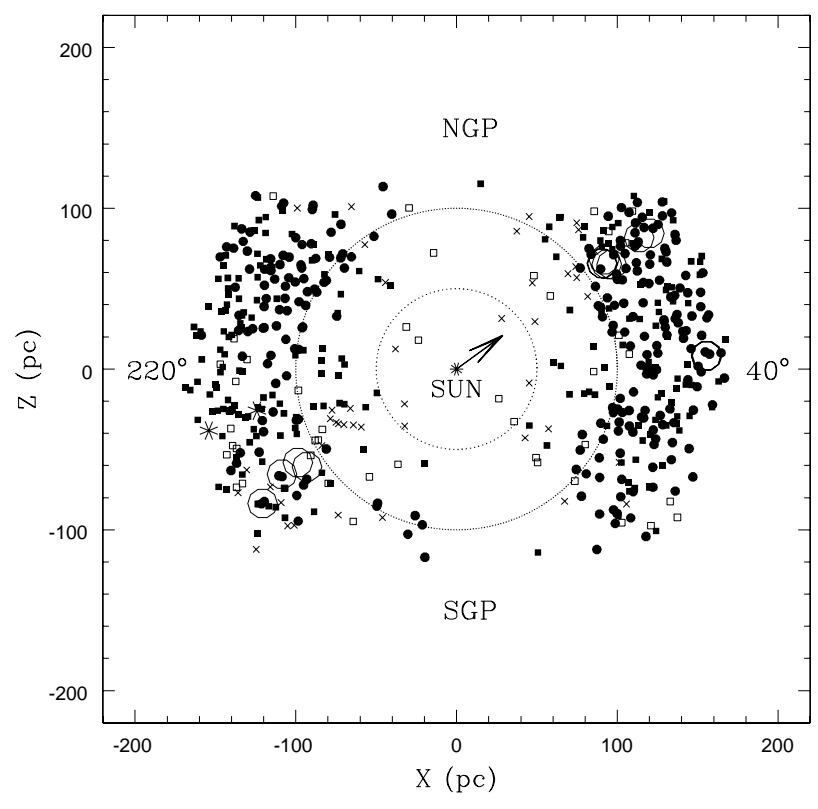

Fig. 5. The distribution of ISM is shown for non-variable stars within $\pm 25^{\circ}$ of a meridian slice perpendicular to the galactic plane and aligned with the solar apex motion through the LSR. The solar apex motion is plotted as an arrow. The $\mathrm{X}$-axis points towards a galactic longitude of $\ell=40^{\circ}$, and the Z-axis points towards the North Galactic Pole. The filled symbols show sightlines with $E(B-V)>0.06 \mathrm{mag}$ (or $\log N(\mathrm{H})>20.48, \mathrm{~cm}^{-2}$ ), and the x's show sightlines with $E(B-V)<0.02$ mag (or $\log N(\mathrm{H})<19.93$, $\left.\mathrm{cm}^{-2}\right)$. Open squares have $E(B-V)=0.02-0.06 \mathrm{mag}$. Large open circles display the positions of nearby dust clouds (see text). These reddening values are determined from photometric data in the Hipparcos catalog (Perryman, 1997). Data points are smoothed over $\pm 13^{\circ}$, and incorporate all stars with overlapping distances (once uncertainties are included). The asterisks indicate the positions of the stars $\epsilon \mathrm{CMa}$ and $\beta \mathrm{CMa}$, which are located in a well-known spatial region with low interstellar densities (Frisch and York, 1983), but which are not included in the plotted star sample because the Hipparcos catalog flags them as variable.

change these entry time values. The star closest to the $\mathrm{HC}$ downwind direction of the LIC $\left(\ell, b \sim 183^{\circ},-16^{\circ}\right)$ is $\chi^{1}$ Ori (HD 39587, at $8.7 \mathrm{pc}$ ). The HC cloud velocity of $21.6 \mathrm{~km} / \mathrm{s}$ towards this star (Redfield and Linsky, 2004a) indicates an entry date into the CLIC of $\sim 56,000$ years ago. We conclude that for no gaps in the ISM distribution within the CLIC, so that $\left\langle n\left(\mathrm{H}^{\circ}\right)\right\rangle \sim 0.17 \mathrm{~cm}^{-3}$ is valid, then the Sun entered the CLIC within the past $\sim 130000 \pm 70000$ years, and possibly within the past 56000 years (consistent with our earlier estimates, Frisch, 1997; Frisch and Slavin, 2006).

If the velocity dispersion of the CLIC clouds represents turbulence, then the assumption of no gaps implies incompressible turbulence. A magnetized partially ionized tenuous plasma will have incompressible turbulence if the magnetic pressure, $\mathrm{P}_{\mathrm{B}}$, is on the order of, or greater than, the thermal pressure, $\mathrm{P}_{\mathrm{TH}}$. Magnetic field strengths of $B \sim 3 \mu \mathrm{G}$ are required in the LIC to balance thermal pressures of $\mathrm{P}_{\mathrm{TH}} \sim 2500 \mathrm{~cm}^{-3} \mathrm{~K}$, as expected from the best photoionization models with $n(\mathrm{e}) \sim 0.1 \mathrm{~cm}^{-3}, \mathrm{H}^{+} \sim 0.1 \mathrm{~cm}^{-3}$, $n\left(\mathrm{H}^{\circ}\right) \sim 0.2 \mathrm{~cm}^{-3}$, and $\mathrm{T}=6300 \mathrm{~K}$. Weaker fields would indicate compressible turbulence and possibly gaps between the cloudlets. In such a case, the entry of the Sun into the CLIC may have occurred earlier than stated above.

The LIC is observed towards both $\alpha \mathrm{CMa}$, at $2.7 \mathrm{pc}$, and $\epsilon$ CMa (towards $\ell \sim 230^{\circ}, b \sim-10^{\circ}$, Hebrard et al., 1999; Gry and Jenkins, 2001). The LIC $N\left(\mathrm{H}^{\circ}\right)$ towards $\alpha \mathrm{CMa}$ has been used to set constraints on the entry date of the Sun into the LIC (Frisch, 1994; Mueller et al., 2006; Frisch and Slavin, 2006). Although the LIC velocity is well known, the cloud structure is uncertain, and we can only state that the Sun first encountered the LIC within the past 40000 years, and possibly within the past 3000 years, based on the earlier discussions.

\section{The Sun in the Local Bubble cavity}

Prior to crossing paths with the CLIC, the Sun traveled through the Local Bubble (LB) for several million years (Frisch and York, 1986). The path of the Sun through the Local Bubble is reconstructed below from reddening data (Sect. 3.1). The LB interior formed one type of paleoLISM, and the limits on the plasma properties of the Local Bubble interior are discussed in Sect. 3.2.

\subsection{Local Bubble}

The exact dimensions and structure of the Local Bubble depend on the ISM component that is sampled; we use the optical reddening properties of interstellar dust grains here, based on photometric data in the Hipparcos catalog (Perryman, 1997), and find a radius of $\sim 60-100$ pc. Earlier maps of the Local Bubble ISM distribution based on reddening data that traces interstellar dust (Lucke, 1978; Perry and Johnston, 1982; Perry and Christodoulou, 1996; Vergely et al., 1997), dust and magnetic fields traced by polarization data (Mathewson and Ford, 1970; Leroy, 1999), and gas traced by $\mathrm{H}^{\circ}$ and $\mathrm{Na}^{\circ}$ (Frisch and York, 1983; Paresce, 1984; Vergely et al., 2001; Lallement et al., 2003) yield similar conclusions about the bubble dimensions, although details of the cavity topology depend on data sensitivity to low column density ISM. Figure 5 shows the motion of the Sun through the Local Bubble, for a plane passing through a meridian, perpendicular to the galactic plane, and aligned with an axis coinciding with the solar apex motion, extending from $\ell=40^{\circ}$ to $220^{\circ}$. Stars with longitudes within $\pm 25^{\circ}$ of this meridian slice are plotted. Cleaned and averaged photometric and astrometric data for $\mathrm{O}, \mathrm{B}$, and A stars in the Hipparcos catalog are used, with variable stars and stars with poorly defined spectral types omitted. 
In tenuous ("intercloud") material, color excess, $E(B-V), \quad$ and $N(\mathrm{H})$ are related by $\log N(\mathrm{H}) / E(B-V)=21.70 \mathrm{~cm}^{-2} \mathrm{mag}^{-1}$ (Bohlin et al., 1978). The CLIC reddening is $E(B-V)<0.002 \mathrm{mag}$, and would not appear on this figure. The large circles show dust clouds from Dutra and Bica (2002). It is evident that for a solar LSR velocity of $13-20 \mathrm{pc} \mathrm{Myrs}^{-1}$, the Sun has been within the very low density Local Bubble for over 3 Myrs. Although CLIC column densities $\left(<10^{19} \mathrm{~cm}^{-2}\right)$ are not traced by typical $E(B-V)$ data, ultraviolet absorption lines do not show any neutral ISM in the anti-apex direction, $\ell \sim 220^{\circ}$, at the $N\left(\mathrm{H}^{\mathrm{o}}\right) \sim 10^{17} \mathrm{~cm}^{-2}$ level between $\sim 5$ and $\sim 100 \mathrm{pc}$ (Frisch and York, 1983, FS06).

\subsection{Local Bubble interior}

We now discuss the properties of the paleolism when the Sun was in the very low density LB interior. The nature and origin of the Local Bubble is the subject of ongoing debate. We expect that this volume is presently filled with a very low density ionized plasma that provides pressure support for the cavity. Observations of the diffuse soft X-ray background (SXRB) led to models of the Local Bubble filled with hot, high pressure gas with a temperature of $\sim 10^{6} \mathrm{~K}$ and pressure of $P / k_{\mathrm{B}} \sim 10^{4} \mathrm{~cm}^{-3} \mathrm{~K}\left(k_{\mathrm{B}}\right.$ is the Boltzman constant), although ROSAT has shown that substantial amounts of the SXRB at high latitude arises beyond the boundaries of the Local Bubble (McCammon et al., 1983; Kuntz and Snowden, 2000). An effective plasma temperature near $10^{6} \mathrm{~K}$ is indicated for a plasma in collisional ionization equilibrium (CIE). Disregarding the problems with models of the emission spectrum, under the assumption that there is a CIE hot plasma filling the LB, the implied density is roughly $5 \times 10^{-3} \mathrm{~cm}^{-3}$ and pressure of $\sim P / k_{\mathrm{B}}=10^{4} \mathrm{~cm}^{-3} \mathrm{~K}$.

There are several implications of the SXRB attributed to the Local Bubble. First, the intensity of the background implies a thermal pressure that is substantially higher than that of the CLIC. This mismatch may be fixed by a CLIC magnetic field of $B \sim 4 \mu \mathrm{G}$. Second, a potentially substantial contribution ( $<30 \%$ in most directions) to the SXRB may arise from charge transfer between solar wind ions such as $\mathrm{O}^{+7}$ and neutral $\mathrm{H}$ or $\mathrm{He}$, either in the interstellar wind or geocorona (Cravens, 2000; Cravens et al., 2001). This does not affect the photoionization calculations described above directly, however, even if the heliospheric and geocoronal soft X-ray emission is at the upper end of current estimates. That is because the heliospheric and geocoronal soft X-ray emission is substantially harder than the emission that is directly responsible for LIC ionization $(\sim 13.6-54.4 \mathrm{eV})$. There is an indirect effect, though, insofar as the heliospheric $\mathrm{X}$-ray emission affects our understanding of the source of the SXRB, which in turn has implications for the lower energy emission and the cloud interface.

This low density hot LB plasma constituted the solar galactic environment for several million years. The LB plasma properties have probably been constant since then, because cooling times for low density plasmas are over $\approx 100$ Myrs. Nevertheless, the LB properties are not constant. Interstellar shocks move through space at velocities over $100 \mathrm{~km} / \mathrm{s}\left(\sim 100 \mathrm{pc} \mathrm{Myrs}^{-1}\right)$, quickly traversing low density ISM. Also, $25 \%$ of the ISM mass is contained in clouds traveling faster than $10 \mathrm{~km} / \mathrm{s}$ through the LSR (based on $\mathrm{H}^{\mathrm{o}}$ 21-cm data in Heiles and Troland, 2003). Therefore, over the past several Myrs some dilution of the plasma must have occurred, particularly near the LB boundaries. The CLIC, with $V_{\mathrm{LSR}} \sim 18 \mathrm{~km} / \mathrm{s}$ may be an example.

The paleoheliosphere formed by the interactions between the very low density plasma of the Local Bubble void and the solar wind has been modeled by Mueller et al. (2006). The resulting heliosphere dimensions are similar to the present heliosphere, but the heliosheath is thicker and pickup ions and other derivatives of interstellar neutrals are absent. These changes have opposite effects on the modulation of galactic cosmic ray fluxes at the Earth.

\subsection{Interface between Local Bubble Plasma and CLIC}

One additional consequence of the presence of the Local Bubble plasma is that a transition region must exist between the tepid CLIC gas and the hot gas. This transition ISM constituted the paleoLISM for a very brief period of time. Models of the interface as a thermally conductive interface indicate a thin region of intermediate temperature gas with the temperature changing by an order of magnitude over a distance of $\sim 1000 \mathrm{AU}$ and more than doubling in the space of $100 \mathrm{AU}$ (Slavin, 1989, SF02). The temperature profile of such a region is very steep near the cloud and flattens farther out as the temperature approaches that of the hot gas. The density profile is similarly shaped, though inverted because the thermal pressure remains nearly constant in the interface. The density increases into the cloud by an order of magnitude over a distance of roughly $1000 \mathrm{AU}$. Over this same distance the ionization is decreasing from nearly complete ionization of $\mathrm{H}$ to the $\sim 30 \%$ of the cloud interior.

The cloud gas is evaporated by the influx of heat and flows off the cloud, accelerated by a gentle pressure gradient created by the thermal conduction. If such a profile exists at the edge of the CLIC gas, the Sun would have traversed from gas at $T \sim 10^{5} \mathrm{~K}$ to $\sim 7000 \mathrm{~K}$ in a period of about 500 years. This would result in a sudden change in the heliospheric boundary conditions that could be very disruptive and result in conditions far from any equilibrium configuration.

An alternative possibility for the boundary is that of a turbulent mixing layer (Slavin et al., 1993). This would be the case if there is a substantial velocity difference between the CLIC gas and that of the hot gas. Even relative velocities on the order of the sound speed in the hot gas or less could be very disruptive to the cooler clouds and could lead to an interface in which the cool gas is being entrained in the hotter gas, mixed and then cooled. Such an interface is similar in 
some ways to the evaporative boundary described above, but depends on hydrodynamic instabilities to create the mixing of the cloud gas into the hot bubble gas. The crossing of this type of interface could also be very disruptive of the heliosphere with the likelihood of small scale condensations and velocity fluctuations as well as sudden variations in ionization in the matter incident on the Solar System.

\section{Discussion}

Simple assumptions are necessary to estimate the eras of the solar transition between galactic environments, although we know that in general the ISM contains a wide range of cloud types and shows coherent structures ranging in size from $<1 \mathrm{pc}$ (e.g. the LIC) to $>100 \mathrm{pc}$ (e.g. Loop I). Another limitation, following from the spectral analysis techniques used to acquire data, is that the identification of an interstellar "cloud" rests on a median velocity determined from the Doppler spread of atomic velocities, which is highly sensitive to the resolution of the instrument conducting the observations (Welty et al., 1994).

Over the ordinary long sightlines of the ISM, e.g. $>100 \mathrm{pc}$, similar simple assumptions are used for clouds that are obviously blended in velocity. The more confined CLIC gas within $\sim 10 \mathrm{pc}$ allows higher levels of accuracy. Howevever, although the electron density diagnostics $\mathrm{Mg}^{\mathrm{O}} / \mathrm{Mg}^{+}$ and $\mathrm{C}^{+*} / \mathrm{C}^{+}$show consistent values of $n(\mathrm{e}) \sim 0.1 \mathrm{~cm}^{-3}$ towards several stars sampling the CLIC, the neutral densities must be reconstructed from radiative transfer models because $\mathrm{C}^{0}$ fine-structure data are unavailable (e.g. Jenkins and Tripp, 2001). Model results indicate consistently that the CLIC does not fill the sightline towards any nearby star, with filling factors of $\sim 0.40$ in the galactic center hemisphere and $\sim 0.26$ in the anti-center hemisphere for $n\left(\mathrm{H}^{\circ}\right) \sim 0.2 \mathrm{~cm}^{-3}$ (Frisch and Slavin, 2006). Except for observations of $\mathrm{Si}^{++}$ towards local stars (e.g. Gry and Jenkins, 2001) and pickup ion $\mathrm{Ne}$, radiative transfer models successfully reproduce densities of ISM towards $\epsilon \mathrm{CMa}$ and within the solar system. Therefore, except for possible hidden dense small clumps of gas, we expect that a cloud density of $n\left(\mathrm{H}^{\circ}\right) \sim 0.2 \mathrm{~cm}^{-3}$ is a reasonable value. Less certain is whether there are gaps in the distribution of the nearest ISM, as would be expected for filamentary LIC gas (Frisch, 1994).

Nevertheless, our understanding of the nearest ISM is not complete, and in particular the origin of the observed macroturbulence indicated by the velocity data (Frisch et al., 2002) and the possibility of denser material in the upwind direction (Frisch, 2003) indicate much of the local ISM remains a mystery. The LIC is our best understood cloud, yet distinctly different estimates of the date of the Sun's entry in the LIC are found depending on which subset of data are used to define an assumed filamentary LIC shape (resulting in entry eras of less than $\sim 10000$ years ago and up to $\sim 40000$ years ago, Frisch, 1994; Mueller et al., 2006). Our analysis here of the paleoLISM provides a starting point for future studies based on improved data and a deeper understanding of turbulence and small-scale structure in the CLIC.

The contribution to the soft X-ray background (SXRB) radiation field at the Sun due to charge exchange between the solar-wind and interstellar neutrals was discussed in Sect. 3.2. The primary effect of this emission will be on the properties of the cloud interface, while the overall soft $\mathrm{X}$-ray flux will continue to be dominated by the strong emission from the Loop I supernova remnant and attenuated extragalactic sources.

\section{Conclusions}

The primary conclusion of this paper is that, over the past several million years, both the galactic environment of the Sun and the heliosphere have been significantly different than they are today. Observational data combined with theoretical studies can be used to reconstruct the three-dimensional distribution of nearby ISM, and predict the times the Sun transitioned between different environments. If we assume a continuously distributed local ISM, within the past $\sim 130000 \pm 70000$ years, and possibly as recent as $\sim 56000$ years ago, the Sun entered low density partially ionized ISM flowing away from the direction of the Scorpius-Centaurus Association. Sometime within the past $\sim 40000$ years the Sun entered the cloud now surrounding the solar system, the LIC. These estimates rely on topologically simple models of the cluster of local interstellar clouds (CLIC) flowing past the Sun; more elaborate models are discussed elsewhere (Frisch, 1994; Gry, 1996; Mueller et al., 2006, FS06). As the Sun moves through this complex of local interstellar clouds, the boundary conditions of the heliosphere should change by substantial amounts due to changes in cloud temperature, velocity, and opacity-driven variations in the ionization of the surrounding ISM. Prior to that, the Sun was in the low density plasma of the Local Bubble cavity. Between the Local Bubble cavity and the CLIC, the Sun briefly ( $\sim 500$ years) passed through an interface region of some type.

These estimates of the entry date of the Sun into the CLIC and LIC are based on current data and models. Future veryhigh resolution observations in the 1000-3000 Å spectral interval, for a spatially dense sample of nearby stars, are required to reconstruct the distribution, kinematics, and properties of the CLIC, and reduce the uncertainties in these estimates. When that happens, we can anticipate that the early work of Hans Fahr will have yielded grand results, as we finally understand the close relationship between the paleoheliosphere and paleoLISM.

Acknowledgements. The authors acknowledge support for this research by NASA grants NAG5-11005, NAG5-13107, NAG513558, and NNG05GD36G. 
Edited by: H.-J. Fahr

Reviewed by: A. Yeghikyan and another referee

\section{References}

Bohlin, R. C., Savage, B. D., and Drake, J. F.: A survey of Interstellar H I from L-alpha Absorption Measurements, Astrophys. J., 224, 132-142, 1978.

Cravens, T. E.: Heliospheric X-ray Emission Associated with Charge Transfer of the Solar Wind with Interstellar Neutrals, Astrophys. J. Lett., 532, L153-L156, 2000.

Cravens, T. E., Robertson, I. P., and Snowden, S. L.: Temporal Variations of Geocoronal and Heliospheric X-ray Emission Associated with the Solar Wind Interaction with Neutrals, J. Geophys. Res., 106, 24 883-24 892, 2001.

Crawford, I. A. and Dunkin, S. K.: Ultra-high-resolution observations of CaII ions in the Local Interstellar Medium, Mon. Not. Roy. Astron. Soc., 273, 219-224, 1995.

Crawford, I. A., Lallement, R., and Welsh, B. Y.: Additional Ultrahigh-resolution observations of CaII ions in the Local Interstellar Medium, Mon. Not. Roy. Astron. Soc., 300, 1181-1188, 1998.

Dehnen, W. and Binney, J. J.: Local Stellar Kinematics from Hipparcos Data, Mon. Not. Roy. Astron. Soc., 298, 387-394, 1998.

Dutra, C. M. and Bica, E.: A Catalogue of Dust Clouds in the Galaxy, Astron. Astrophys., 383, 631-635, 2002.

Fahr, H. J.: On the Influence of Neutral Interstellar Matter on the Upper Atmosphere, Astrophys. Space Sci., 2, 474-495, 1968.

Fahr, H. J.: The Extraterrestrial UV-Background and the Nearby Interstellar Medium, Space Science Rev., 15, 483-540, 1974.

Fahr, H. J.: Change of Interstellar Gas Parameters in Stellar-winddominated astrospheres Solar case, Astron. Astrophys., 66, 103117, 1978.

Ferland, G. J., Korista, K. T., Verner, D. A., Ferguson, J. W., Kingdon, J. B., and Verner, E. M.: CLOUDY 90: Numerical Simulation of Plasmas and Their Spectra, Pub. Astron. Soc. Pacific, 110, 761-778, 1998.

Ferlet, R., Vidal-Madjar, A., and Lallement, R.: The Local Interstellar Medium in the Direction of Alpha Aquilae, Astron. Astrophys., 163, 204-209, 1986.

Florinski, V., Zank, G. P., and Axford, W. I.: The Solar System in a dense interstellar cloud: Implications for Cosmic-ray Fluxes at Earth and ${ }^{10}$ Be records, Geophys. Res. Lett., 30, 5-1, $2003 \mathrm{a}$.

Florinski, V., Zank, G. P., and Axford, W. I.: The Solar System in a Dense Interstellar Cloud: Implications for Cosmic-Ray Fluxes at Earth and ${ }^{10} \mathrm{Be}$ records, Geophys. Res. Lett., 30, 5-1, 2003b.

Frisch, P. and York, D. G.: Interstellar Clouds Near the Sun, in: The Galaxy and the Solar System, Univ. of Arizona Press, 83-100, 1986.

Frisch, P. C.: The Nearby Interstellar Medium, Nature, 293, 377379,1981

Frisch, P. C.: G-Star Astropauses - A Test for Interstellar Pressure, Astrophys. J., 407, 198-206, 1993.

Frisch, P. C.: Morphology and Ionization of the Interstellar Cloud Surrounding the Solar System, Science, 265, 1423, 1994.

Frisch, P. C.: Characteristics of Nearby Interstellar Matter, Space Sci. Rev., 72, 499-592, 1995.

Frisch, P. C.: Journey of the Sun, http://xxx.lanl.gov/astro-ph/ 9705231, 1997.
Frisch, P. C.: Local Interstellar Matter: The Apex Cloud, Astrophys. J., 593, 868-873, 2003.

Frisch, P. C.: Introduction: The Paleoheliosphere versus PaleoLISM, in Solar Journey: The Significance of Our Galactic Environment for the Heliosphere and Earth, edited by Frisch, P. C., in press, Springer, 2006.

Frisch, P. C. and Slavin, J. D.: Short Term Variations in the Galactic Environment of the Sun, in press, 2006.

Frisch, P. C. and York, D. G.: Synthesis Maps of Ultraviolet Observations of Neutral Interstellar Gas, Astrophys. J. Lett., 271, L59-L63, 1983.

Frisch, P. C., Grodnicki, L., and Welty, D. E.: The Velocity Distribution of the Nearest Interstellar Gas, Astrophys. J., 574, 834846, 2002.

Gry, C.: Local Clouds : Distribution, Density and Kinematics through Ground-Based and HST Spectroscopy, Space Sci. Rev., 78, 239-246, 1996.

Gry, C. and Jenkins, E. B.: Local clouds: Ionization, Temperatures, Electron Densities and Interfaces, from GHRS and IMAPS Spectra of $\epsilon$ Canis Majoris, Astron. Astrophys., 367, 617-628, 2001.

Hébrard, G. and Moos, H. W.: The Deuterium-to-Oxygen Ratio in the Interstellar Medium, Astrophys. J., 599, 297-311, 2003.

Hebrard, G., Mallouris, C., Ferlet, R., Koester, D., Lemoine, M., Vidal-Madjar, A., and York, D.: Ultraviolet observations of Sirius A and Sirius B with HST-GHRS. An interstellar cloud with a possible low deuterium abundance, Astron. Astrophys., 350, 643-658, 1999.

Heiles, C. and Troland, T. H.: The Millennium Arecibo 21 Centimeter Absorption-Line Survey. I. Techniques and Gaussian Fits, Astrophys. J. Suppl., 145, 329-354, 2003.

Jenkins, E. B. and Tripp, T. M.: The Distribution of Thermal Pressures in the Interstellar Medium from a Survey of C I FineStructure Excitation, Astrophys. J. Suppl., 137, 297-340, 2001.

Kruk, J. W., Howk, J. C., André, M., Moos, H. W., Oegerle, W. R., Oliveira, C., Sembach, K. R., Chayer, P., Linsky, J. L., Wood, B. E., Ferlet, R., Hébrard, G., Lemoine, M., VidalMadjar, A., and Sonneborn, G.: Abundances of D, N and O toward HZ 43A: Results from the FUSE Mission, Astrophys. J. Suppl., 140, 19-36, 2002.

Kuntz, K. D. and Snowden, S. L.: Deconstructing the Spectrum of the Soft X-Ray Background, Astrophys. J., 543, 195-215, 2000.

Lallement, R., Welsh, B. Y., Vergely, J. L., Crifo, F., and Sfeir, D.: 3-D Mapping of the Dense Interstellar Gas around the Local Bubble, Astron. Astrophys., 411, 447-464, doi:10.1051/ 0004-6361:20031214, 2003.

Lehner, N., Jenkins, E., Gry, C., Moos, H., Chayer, P., and Lacour, S.: FUSE Survey of Local Interstellar Medium within 200 Parsecs, Astrophys. J., 595, 858-879, 2003.

Lemoine, M., Vidal-Madjar, A., Hébrard, G., Désert, J.-M., Ferlet, R., Lecavelier des Étangs, A., Howk, J. C., André, M., Blair, W. P., Friedman, S. D., Kruk, J. W., Lacour, S., Moos, H. W., Sembach, K., Chayer, P., Jenkins, E. B., Koester, D., Linsky, J. L., Wood, B. E., Oegerle, W. R., Sonneborn, G., and York, D. G.: Deuterium Abundance toward G191-B2B: Results from the FUSE Mission, Astrophys. J. Suppl., 140, 67-80, 2002.

Leroy, J. L.: Interstellar dust and magnetic field at the boundaries of the Local Bubble. Analysis of polarimetric data in the light of HIPPARCOS parallaxes, Astron. Astrophys., 346, 955-960, 
1999.

Lucke, P. B.: The Distribution of Color Excesses and Interstellar Reddening Material in the Solar Neighborhood, Astron. Astrophys., 64, 367-377, 1978.

Möbius, E., Bzowski, M., Chalov, S., Fahr, H.-J., Gloeckler, G., Izmodenov, V., Kallenbach, R., Lallement, R., McMullin, D., Noda, H., Oka, M., Pauluhn, A., Raymond, J., Ruciński, D., Skoug, R., Terasawa, T., Thompson, W., Vallerga, J., von Steiger, R., and Witte, M.: Synopsis of the Interstellar He Parameters from Combined Neutral Gas, Pickup Ion and UV Scattering Observations, Astron. Astrophys., 426, 897-907, 2004.

Mathewson, D. S. and Ford, V. L.: Polarization Observations of 1800 Stars, Mem. Roy. Astron. Soc., 74, 139-182, 1970.

McCammon, D., Burrows, D. N., Sanders, W. T., and Kraushaar, W. L.: The Soft X-ray Diffuse Background, Astrophys. J., 269, 107-135, 1983.

Mihalas, D. and Binney, J.: Galactic Astronomy, Freeman, San Francisco, 1981.

Mueller, H. R., Frisch, P. C., Florinski, V., and Zank, G. P.: Heliospheric Response to Different Possible Interstellar Environments, Astrophys. J., in press, 2006.

Oliveira, C. M., Hébrard, G., Howk, J. C., Kruk, J. W., Chayer, P., and Moos, H. W.: Interstellar D, N and $\mathrm{O}$ Abundances toward GD 246, WD 2331-475, HZ 21, and Lanning 23: Results from the FUSE Mission, Astrophys. J., 587, 235-255, 2003.

Paresce, F.: On the Distribution of Interstellar Matter around the Sun, Astron. J., 89, 1022-1037, 1984.

Pepino, R., Kharchenko, V., Dalgarno, A., and Lallement, R.: Spectra of the X-Ray Emission Induced in the Interaction between the Solar Wind and the Heliospheric Gas, Astrophys. J., 617, 13471352, 2004.

Perry, C. L. and Christodoulou, D. M.: Interstellar Reddening in the Southern Hemisphere. II. Analysis of the uvby-Beta Observations, Pub. Astron. Soc. Pacific, 108, 772-816, 1996.

Perry, C. L. and Johnston, L.: A Photometric Map of Interstellar Reddening within 300 parsecs, Astrophys. J. Suppl., 50, 451$515,1982$.

Perryman, M. A. C.: The HIPPARCOS Catalogue, Astron. Astrophys., 323, L49-L52, 1997.

Redfield, S. and Linsky, J. L.: Microstructure of the Local Interstellar Cloud and the Identification of the Hyades Cloud, Astrophys. J., 551, 413-428, 2001.

Redfield, S. and Linsky, J. L.: The Structure of the Local Interstellar Medium. II. Observations of D I, C II, N I, O I, Al II, and Si II toward Stars within 100 Parsecs, Astrophys. J., 602, 776-802, 2004a.

Redfield, S. and Linsky, J. L.: The Structure of the Local Interstellar Medium. III. Temperature and Turbulence, Astrophys. J., 613, 1004-1022, 2004b.

Ripken, H. W. and Fahr, H. J.: Modification of the Local Interstellar Gas Properties in the Heliospheric Interface, Astron. Astrophys., 122, 181-192, 1983.

Rogerson, J. B., York, D. G., Drake, J. F., Jenkins, E. B., Morton, D. C., and Spitzer, L.: Spectrophotometric Results from the Copernicus Satellite. III. Ionization and Composition of the Intercloud Medium, Astrophys. J. Lett., 181, L110-L114, 1973.

Scherer, K.: Variation of the heliospheric shield and its influence on Earth, in: The outer heliosphere: beyond the planets, edited by Scherer, K., Fichtner, H., and Marsch, E., Katlenburg-Lindau,
Germany: Copernicus-Gesellschaft, ISBN 3-9804862-3-0, 327$355,2000$.

Scherer, K. and Fahr, H. J.: Breathing of Heliospheric Structures Triggered by the Solar-Cycle Activity, Ann. Geophysicae, 21, 1303-1313, 2003.

Scherer, K., Fichtner, H., and Stawicki, O.: Shielded by the Wind: The Influence of the Interstellar Medium on the Environment of Earth, J. Atmos. Terr. Phys., 64, 795-804, 2002.

Slavin, J. D.: Consequences of a Conductive Boundary on the Local Cloud. I - No Dust, Astrophys. J., 346, 718-727, 1989.

Slavin, J. D. and Frisch, P. C.: The Ionization of Nearby Interstellar Gas, Astrophys. J., 565, 364-379, 2002.

Slavin, J. D., Shull, J. M., and Begelman, M. C.: Turbulent Mixing Layers in the Interstellar Medium of Galaxies, Astrophys. J., 407, 83-99, 1993.

Vallerga, J.: Observations of the Local Interstellar Medium with the Extreme Ultraviolet Explorer, Space Sci. Rev., 78, 277-288, 1996.

Vergely, J.-L., Egret, D., Freire Ferrero, R., Valette, B., and Koeppen, J.: The Extinction in the Solar Neighbourhood from the HIPPARCOS Data, in: ESA SP-402: Hipparcos - Venice 97, 402, 603-606, 1997.

Vergely, J.-L., Freire Ferrero, R., Siebert, A., and Valette, B.: NaI and HI 3-D Density Distribution in the Solar Neighbourhood, Astron. Astrophys., 366, 1016-1034, 2001.

Welty, D. E., Hobbs, L. M., and Kulkarni, V. P.: A High-resolution Survey of Interstellar Na I D1 lines, Astrophys. J., 436, 152-175, 1994.

Welty, D. E., Morton, D. C., and Hobbs, L. M.: A High-Resolution Survey of Interstellar Ca II Absorption, Astrophys. J. Suppl., 106, 533-562, 1996.

Witte, M.: Kinetic Parameters of Interstellar Neutral Helium. Review of Results Obtained During One Solar Cycle with the Ulysses/GAS-instrument, Astron. Astrophys., 426, 835-844, 2004.

Wolff, B., Koester, D., and Lallement, R.: Evidence for an Ionization Gradient in the Local Interstellar Medium: EUVE Observations of White Dwarfs, Astron. Astrophys., 346, 969-978, 1999.

Wood, B. E., Linsky, J. L., Hébrard, G., Vidal-Madjar, A., Lemoine, M., Moos, H. W., Sembach, K. R., and Jenkins, E. B.: Deuterium Abundance toward WD 1634-573: Results from the FUSE Mission, Astrophys. J. Suppl., 91-102, 2002.

Wood, B. E., Redfield, S., Linsky, J. L., Müller, H.-R., and Zank, G. P.: Stellar Ly $\alpha$ Emission Lines in the Hubble Space Telescope Archive: Intrinsic Line Fluxes and Absorption from the Heliosphere and Astrospheres, Astrophys. J. Suppl., 159, 118-140, 2005.

Yeghikyan, A. and Fahr, H.: Effects Induced by the Passage of the Sun through Dense Molecular Clouds. I. Flow Outside of the Compressed Heliosphere, Astron. Astrophys., 415, 763-770, 2004.

York, D. G.: Lambda Sco, Astrophys. J., 264, 172-195, 1983.

Zank, G. P. and Frisch, P. C.: Consequences of a Change in the Galactic Environment of the Sun, Astrophys. J., 518, 965-973, 1999. 